\title{
Performance Evaluation of Four Grid-Forming Control Techniques with Soft Black-Start Capabilities
}

\author{
Abdulrahman Alassi ${ }^{1,2, *}$, Student Member, IEEE, Khaled Ahmed ${ }^{2}$, Senior Member, IEEE, Agusti Egea-Alvarez ${ }^{2}$, Member, IEEE \\ and Omar Ellabban ${ }^{1}$, Senior Member, IEEE. \\ ${ }^{1}$ Iberdrola Innovation Middle East, Doha, Qatar, *aalassi@iberdrola.com \\ ${ }^{2}$ Department of Electronic and Electrical Engineering, University of Strathclyde, Glasgow, UK.
}

\begin{abstract}
Grid-Forming Converters (GFC) can be controlled as independent, self-starting, voltage sources. This feature is essential for power converters to achieve successful black-start sequence initiation. Conventional grid-following converters are not capable of self-starting an islanded network. GFC control thus exploits wider grid support and network restart potential. This study analyzes and compares four GFC controllers to assess their generic and soft black-start (ramping voltage) capabilities. The compared techniques are: Droop Control, Power Synchronizing Control (PSC), Virtual Synchronous Machine (VSM), and Matching control. These techniques are selected based on their direct voltage reference control flexibility. Various simulations are performed with common parameters to assess the response of each technique under similar conditions against load, DC voltage and active power reference disturbances, in addition to their soft-start readiness. The results demonstrate the high-level compatibility of these four controllers with soft black-start through successful and timely ramping voltage reference tracking. Moreover, the four considered control techniques achieve satisfactory performance, with VSM demonstrating more flexibility due to its tunable virtual inertia parameter $(J)$.
\end{abstract}

Keywords-Grid-Forming Converters, Black-Start, Soft-Start, Droop, VSM, PSC, Matching Control, MATLAB/Simulink.

\section{INTRODUCTION}

The increased renewable energy sources (RES) and distributed energy resources (DER) penetration through voltage source converters (VSCs) has been contributing to a change in the classical network-operating paradigm that relied on synchronous machines (SMs) dominated grids [1-3]. VSCs gridconnection can be achieved in grid-following or grid-forming control modes. The former has been long used as an industrial standard for renewables grid integration. Though, the participation of grid-following controlled VSCs in networkrestoration after a system blackout is restricted and requires an external source to energize first in order for them to "follow" [4]. Grid-following RES converters are required by different gridcodes to disconnect during network restoration to alleviate any additional problems that may result from their output intermittency $[4,5]$. On the other hand, grid-forming converters (GFCs) can establish an independent voltage, which is suitable for black-start initiation and various grid-support applications [6]. Generally, converter-connected RES/DER with topologies that possess the flexibility to control the DC link voltage at the grid-side converter input (e.g., coupled PV and battery storage or Type IV wind turbines) can be used in grid-forming mode.

Although extremely rare, the occurrence of a system-level cascaded blackout can directly or indirectly cost the network operators and customers millions of dollars [7]. Two main approaches that utilize existing network assets can be followed to preserve system strength and the network black-start readiness as a result of the new network status: (a) maintaining the operation of existing SMs, regardless of their demand offset by renewables, which could lead to increased operational cost as reported in [4]. (b) operating the grid-following converters in grid-forming mode, as independent voltage sources that can reestablish the electric island power network. The latter alternative can also be combined with auxiliary synchronous condensers in the network bottlenecks in case the network short circuit ratio (SCR) value becomes critically low [6]. Network restoration involves the energization of transformers and lines, which typically require high inrush currents that may exceed several times the converter rating [8]. Many SMs can withstand the resulting short-term overcurrent, but converters are more sensitive to such conditions. Therefore, soft-start (ramping voltage energization) is suggested as an alternative to mitigate inrush currents in case converters are to act as primary (anchor) energizing sources during network restoration events.

Many works in literature proposed and analyzed GFCs for different applications such as inertia support, grid-connected and islanded microgrids operation, ancillary services provision to the grid, and more $[1,6]$. Though, the number of published studies investigating their use for black-start applications has been limited without detailed scope from the control point of view, with some reported industrial-scale tests $[9,10]$. On the other hand, several classifications of GFCs have been introduced, such as classifying grid-forming control techniques into inertial and non-inertial, or converter DC side vs. AC side reliant. For a black-start scenario, an important classification can also be based on the technique compatibility with a direct voltage reference control to be able to use a ramping reference, as not all techniques inherently provide this flexibility. An example of such technique (in its basic form) is the inducverter controller, first proposed in [11].

References $[2,12,13]$ each investigated the capabilities, performance and market maturity of different grid forming techniques and provide a good summary of their merits. The considered techniques in these works are: droop control, Power Synchronizing Control (PSC), dispatchable Virtual Oscillator Control (dVOC), matching control, and Virtual Synchronous Machine (VSM). The authors of [2] highlight that a GFC selection for a satisfactory AC side performance (i.e., Rate-ofChange-of-Frequency (RoCoF) and frequency nadir reduction) may not necessarily yield similar results for operating the DC side source near its saturation limits. This shows the necessity of studying the often-neglected coupled reaction between the DC and $\mathrm{AC}$ side dynamics to achieve a global control objective that maintains stability against disturbances in both sides of 


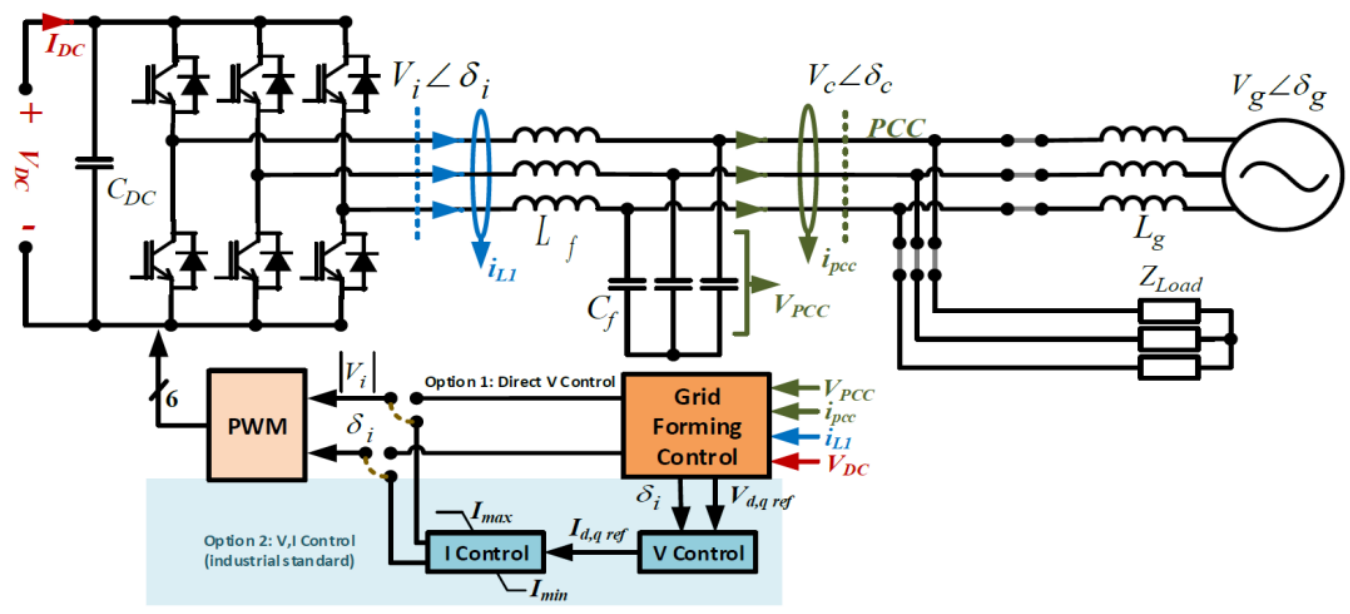

Fig. 1. High-level control block diagram of a 2-levels,3-phase VSC based GFC.

operation. The authors of [12] present a comparison from transient stability design point of view for inertial and noninertial GFCs. Finally, the work presented in [13] offers a highlevel summary of droop, VSM, dVOC and matching GFCs principle of operation and market maturity.

Based on the different existing classifications, this paper will investigate four different GFC control techniques using the network topology in Fig. 1 to evaluate their performance under grid-connected and high-level black-start scenarios. The conducted tests will assess the controllers performance against different $\mathrm{AC}$ and $\mathrm{DC}$ disturbances, and their high-level suitability for black-start applications through soft-start voltage ramps reference tracking. The four compared GFC control techniques are: droop, PSC, VSM and matching control. The selection for these four techniques is justified as below:

1. Droop and PSC are selected due to the wide use of the former, and the similarities between both techniques [12, 14].

2. VSM control performs best in terms of AC side disturbances study, whereas matching control performs best against DC side source saturation in [2].

3. dVOC is disqualified from this comparison due to its moderate performance against both disturbance types in [2].

Consequently, the contributions of this manuscript are summarized as follows:

1. Testing each controller under different operating conditions and disturbances to verify their performance patterns.

2. Identifying the suitable GFC controllers for black-start applications with inherent soft-start capability.

3. Testing and verifying the stable GFC controllers operation under a simplified soft-start operating scenario.

\section{Testing Methodology}

The testing methodology aims at targeting each control technique individually and to measure its response under various operating conditions based on Fig. 1 configuration. The four GFC controllers are tested under a unified steady state powerfrequency slope to mitigate the impact of control design variations. The common steady state power-frequency slope is estimated for all techniques as in (1).

$$
\omega_{\text {ref }}-\omega=d_{s}\left(P-P_{\text {ref }}\right)
$$

where, $d_{s}$ is the slope that is unified among the different techniques for consistency, $\omega$ and $\omega_{\text {ref }}$ are the measured and reference angular frequencies, respectively, with a similar analogy for the active power parameters $P$ and $P_{\text {ref }}$. The selected value of $d_{s}$ is such that a $100 \%$ swing in rated power results in $1 \%$ swing in frequency (i.e., $49.5 \mathrm{~Hz}-50.5 \mathrm{~Hz}$ ).

Dual loop voltage and current control is implemented with similar PI tuned gains for all techniques $\left(K p_{v}=0.1, K i_{v}=\right.$ $\left.1, K p_{i}=9, K i_{i}=0.287\right)$. Moreover, a dynamic DC link control is implemented rather than a stiff DC voltage source since matching control relies on changes in DC link voltage for its power-frequency synchronizing function. The same DC link voltage control is applied for all techniques for consistency based on [2]. The test system rating is representative of a realistic power plant connected to a $33 \mathrm{kV}$ bus. The DC link and the grid converter $\mathrm{AC}$ output are rated at their nominal value under the assumption of simplified connections (direct load energization). The test system parameters are summarized in Table 1. A network is classified as weak if it has a Short-Circuit-Ratio $(\mathrm{SCR})<3$ [15], and thus an intermediate value of $\mathrm{SCR}=5$ is selected, whereas the network $\mathrm{X} / \mathrm{R}$ ratio is selected as 10 to reflect the upper range from [16]. MATLAB/Simulink environment is used to simulate the network and study the performance of each considered GFC controller.

The considered tests for all techniques are: a) load disturbance at the Point-of-Common-Coupling (PCC) with $P=$ 1 pu. b) DC link voltage step increase by $0.2 p u, c)$ active power reference disturbance from $0.5 \mathrm{pu}$ to $1 \mathrm{pu}$. d) soft voltage ramp tracking from 0 to $1 \mathrm{pu}$ for each technique in 10 seconds to track a $35 \mathrm{MW}, 5 \mathrm{MVAr}$ load, emulating a high-level soft black-start scenario. The impact of tests (a)-(c) is measured on frequency, RoCoF, active power, PCC voltage and DC voltage to identify the techniques robustness, whereas test (d) verifies the voltage and frequency tracking capability in the soft-startup scenario. Finally, the controllers response is numerically evaluated using a Performance Ratio (PR) parameter, whereby the best performing technique in each category (e.g., lowest frequency nadir) is assigned a value of 1 , and the performance of other GFCs is measured in terms of its PR against this value as in (2), 
Table 1. GFC Test system parameters

\begin{tabular}{cccc}
\hline PCC Voltage (LL-kV) & 33 & Converter Rating (MVA) & 36 \\
DC Link Voltage (kV) & 54 & Network XR Ratio & 10 \\
Active Power (MW) & 35 & Short-Circuit-Ratio (SCR) & 5 \\
Nominal Frequency (Hz) & 50 & Reactive Power (MVAr) & 5 \\
\hline
\end{tabular}

where $G F C_{s S}$ is the measured parameter at the pre-disturbance steady state (e.g., 1 pu for frequency and 0 for RoCoF), $G F C_{i}$ is the $i^{\text {th }}$ technique metric, and the $G F C_{\text {best }}$ is the best performing controller metric for the measured parameter.

$$
P R=\frac{G F C_{S S}-G F C_{i}}{G F C_{S S}-G F C_{\text {best }}}
$$

\section{INVESTIGATED GRID-FORMING CONTROLLERS}

\section{A. Droop Control}

Droop is a common technique that is used for several converter control applications, especially when power sharing between various VSCs is required in a similar fashion to that of SMs. A fully optimized droop implementation for VSC applications is still an active research question that attracts research to overcome performance limitations [3].

Conventional droop control under inductive network assumption includes a P-f loop and a Q-V loop. The former loop is responsible for frequency deviation and angle reference generation (Fig. 2a), while the latter is responsible for voltage and reactive power regulation (Fig. 2b). From the different possible implementations, the PCC voltage tracking path is adopted in this work. $K_{P}$ and $K_{q}$ are the power and reactive power droop coefficients, respectively, and are defined as in (3).

$$
\begin{aligned}
& K_{P}=-\frac{\omega_{r e f}-\omega}{P_{r e f}-P} \\
& K_{q}=-\frac{V_{r e f}-V}{Q_{r e f}-Q}
\end{aligned}
$$

where, $V_{\text {ref }}$ and $V$ are the nominal and measured AC side voltages, respectively.

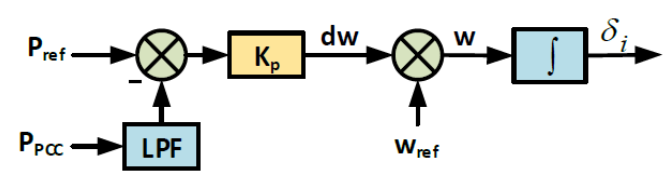

(a)

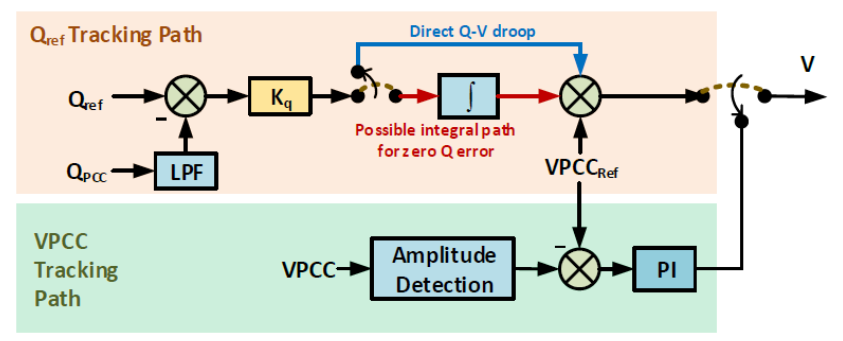

(b)

Fig. 2. Droop control block diagram: (a) Power loop, (b) Voltage loop with various implementations.

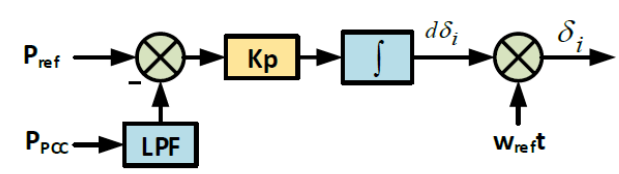

Fig. 3. PSC Controller - Power Synchronizing Loop.

\section{B. Power Synchronizing Control}

This VSC control technique was first introduced in [14], mainly for HVDC applications connected to weak grids in order to mitigate the stability issues of PLLs. The proposed controller includes a Power Synchronizing Loop (PSL), in addition to voltage and reactive power control loops. These loops are connected or disconnected depending on the application needs. A backup PLL is proposed in the original design to provide synchronization under fault conditions. The voltage/reactive power loops are similar in nature to those discussed in the droop control section. Thus, the main studied variation is in the PSL loop, which is illustrated in Fig. 3.

\section{Virtual Synchronous Machine (VSM)}

The used terminology for this technique might differ between various papers such as synchronverter or VSM based on the implementation. The term "VSM" is used in this paper context to refer to the technique introduced in [17]. This control concept stems from SMs characteristics emulation. Most existing implementations focus on emulating the SM inertial response to frequency events through the SM swing equation. In contrast to the other techniques discussed here, VSM power control loops include additional term(s), which emulate frequency dynamics through $d \omega / d t$ into the control design as in equation (4).

$$
J \frac{d \omega}{d t}=\frac{1}{\omega_{\text {ref }}}\left(P_{\text {ref }}-P\right)-D_{p}\left(\omega_{\text {ref }}-\omega\right)
$$

where, $J$ is the virtual moment of inertia, and $D_{p}$ is the damping factor. The value of $J$ influences the rate of change of frequency (RoCoF) and its minimum/maximum (nadir/zenith) value in case of a frequency event. The virtual inertia constant $J$ can be considered as an additional independent degree of freedom in VSM design, which can be optimized for enhanced performance. On the other hand, VQ tracking are both present in the conventional VSM voltage loop implementation. The conventional VSM implementation is presented in Fig. 4. The VQ loop is slightly modified in this paper to prioritize the PCC voltage tracking objective. $M_{f}$ analogy is inspired from the mutual inductance between a SM field and stator coils, and $i_{f}$ is inspired from the rotor excitation current. The parameter $1 / K_{v}$ is the voltage loop integrator gain, and $D_{q}$ is the voltage drooping coefficient.

\section{Matching Control}

This grid-forming control technique has recently been introduced in [18], similarly aiming to exploit structural similarities between VSCs and SMs. The matching control principle is based on the observation that the DC link voltage variations indicate power imbalances in an analogy to SMs 


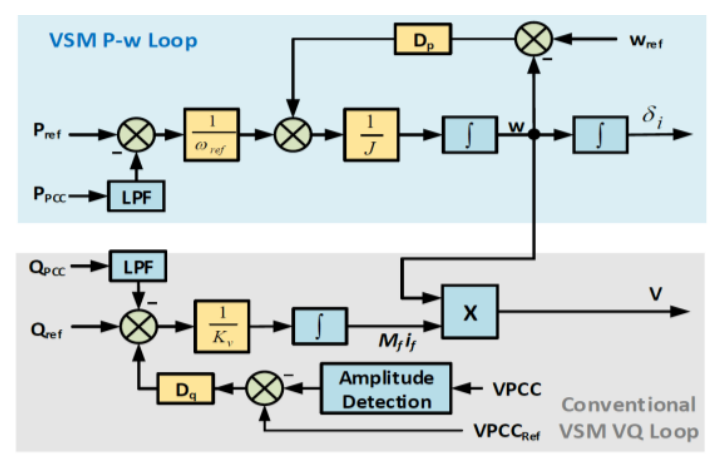

Fig. 4. VSM control block with conventional P/V loops.

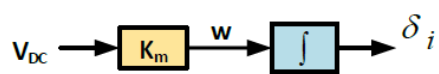

Fig. 5. Matching control Power loop, based on DC voltage variations.

frequency, this analogy is discussed in more details in [19]. The power loop uses this analogy to drive the converter frequency from the dynamic DC link voltage as illustrated in Fig. 5.

The matching control power loop is fairly simple, as it mainly transforms the DC link voltage to a converter frequency signal using the transformation factor $K_{m}$ defined in (5), which is then integrated to generate the converter synchronizing angle.

$$
K_{m}=\frac{\omega_{r e f}}{V_{D C_{r e f}}}
$$

The voltage loop in matching control also has various implementations in literature (including a similar one to Fig. 2b, adopted here). The voltage magnitude control output factor in this technique can be derived to meet various control objectives such as PCC voltage regulation as in [2] or active/reactive power tracking as in [20].

\section{SimUlATION RESUlTS}

\section{A. Load Disturbance Response: 1 pu load connection at PCC}

The four controllers response to this disturbance is similar in terms of magnitude changes in the tested parameters. Fig. 6 parts (a) to (e) summarize the four GFC responses to this disturbance, illustrating satisfactory performance for all of them, considering their small pu variations for short durations. Fig. 6f then summarizes the GFCs performance comparison against load disturbance for the frequency nadir, RoCoF and the converter active power step change using the established PR parameter in (2). The main step variation among the five measured parameters for this disturbance occurs in the PCC voltage, with a magnitude change approaching $10 \%$ of nominal value, though, only for few milliseconds. This considerable change is due to the sudden load connection at the PCC.

\section{B. DC Voltage Disturbance Response: 1 pu to $1.2 \mathrm{pu}$}

Three of the four techniques had nearly neutral response to this type of disturbance, except for matching control as it depends on DC voltage in its control. The analysis presented in [2] demonstrated an enhanced performance for matching control against DC current operating near its source saturation, whereas the disturbance applied here measures the controller response against a disturbance in the DC link voltage (energy buffer) under unsaturated conditions. The reported frequency nadir for all techniques is well within grid requirements with the minimum value in matching control corresponding to 0.997 pu (although relatively way higher than the other techniques). Similar behavior is observed for RoCoF (0.209 pu, matching), and maximum active power variation (0.17 pu, matching). Similarly, Fig. 7 summarizes the results of this test.

\section{Pref Disturbance Response: 0.5 pu to 1 pu}

This disturbance represents a $0.5 \mathrm{pu}$ active power reference step for the grid connected converter (from $P=0.5$ pu to $P=1$ $p u)$. The maximum variation magnitude for the main parameters is $0.0059 \mathrm{pu}$ for frequency zenith (Droop), $0.25 \mathrm{pu}$ for RoCoF (Droop), 1.042 pu maximum power step (VSM). Minimal variations are also observed for DC link and PCC voltages. Clearly, the measured variations are minimal and similarly demonstrate satisfactory performance by the different controllers against this disturbance. It should be noted that unlike previous tests, the frequency and RoCoF in this case are in the positive direction since the converter has to increase its output power to a new steady state reference. Finally, Fig. 8 summarizes the results of this test in a similar fashion.

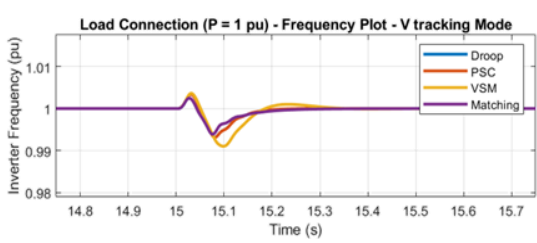

(a)

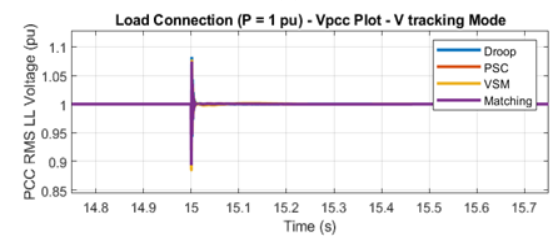

(d)

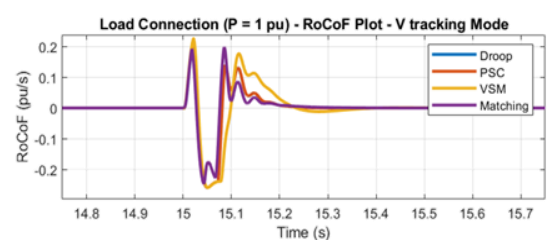

(b)

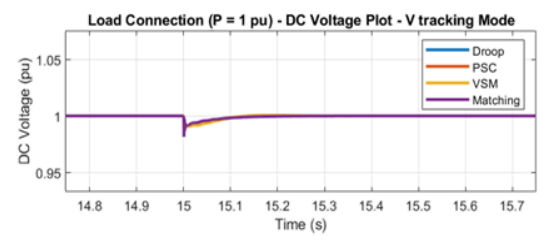

(e)

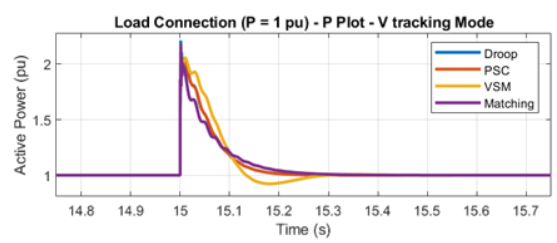

(c)

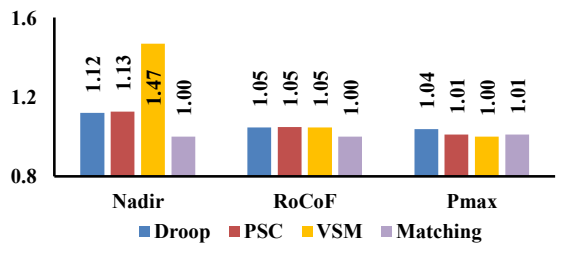

(f)

Fig. 6. GFCs control response to the load disturbance scenario. (a) Frequency plot, (b) RoCoF plot, (c) active power plot, (d) PCC voltage plot, (e) DC link voltage plot, (f) statistical GFC responses comparison. 


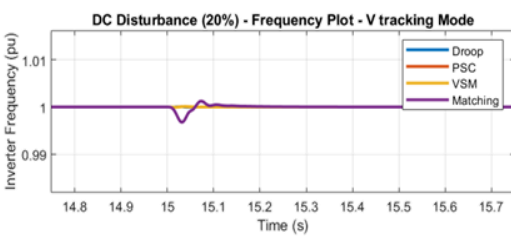

(a)

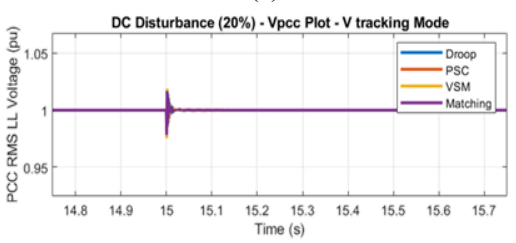

(d)

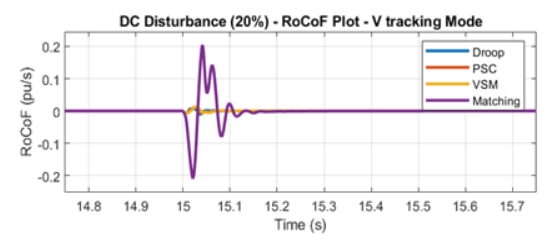

(b)

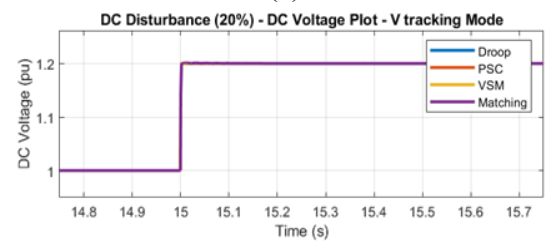

(e)

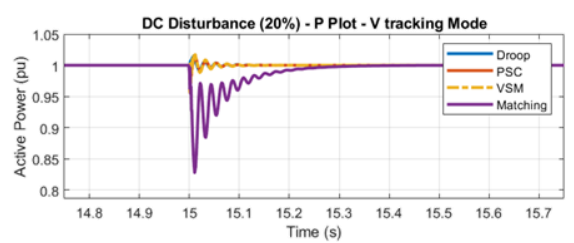

(c)

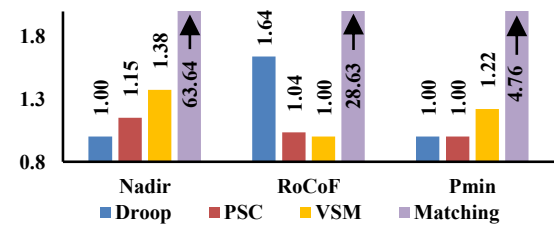

(f)

Fig. 7: GFCs response to the DC voltage disturbance scenario. (a) Frequency plot, (b) RoCoF plot, (c) active power plot, (d) PCC voltage plot, (e) DC voltage plot, (f) statistical GFC responses comparison.

\section{Soft Black-Start Test}

This test aims to verify the soft black-start capability of the tested controllers on a high, reference-tracking, level. Depending on the operating mode, some techniques synthesize the $\mathrm{AC}$ voltage reference through $\mathrm{P} / \mathrm{PI}$ controllers to convert the PCC voltage error into a reference. The speed at which this voltage reference is synthesized depends on the selected controller gains. Soft-start is typically implemented to ramp up the voltage to a reference at a specific slope based on the network requirements (e.g., within 10 seconds in this test), and thus the P/PI control design should take into consideration having a minimal impact on the desired ramping action.

As the reactive power requirement during black-start can be varying with a level of uncertainty, a PCC voltage tracking control mode is employed in this test scenario, where $\mathrm{P}=35$ $\mathrm{MW}$ and $\mathrm{Q}=5 \mathrm{MVAr}$ loads from Table 1 are connected in an islanded network configuration and the voltage ramp is observed for all controllers. Fig. 9 summarizes the test results and illustrates successful PCC voltage tracking from 0 to $1 \mathrm{pu}$ with no measurable delays from $t=0$ to $t=10 \mathrm{~s}$, as well as for the active and reactive power references for all controllers. The frequency in Fig. 9c descends from an initial value to $1 \mathrm{pu}$ at $t=10 \mathrm{~s}$ as a result of the initial power reference mismatch.

\section{E. Results Discussion}

The conducted tests reveal that the four controllers with current control perform similarly against the three considered power and voltage disturbances. Voltage tracking objective for the VQ loop is assumed in all cases since it is more relevant to a black-start scenario with volatile reactive power probability. The same three disturbances in Section IV(A)-(C) were applied separately to the four controllers with comparable responses. Droop and PSC performed almost equally due to their structural similarities. Matching control demonstrated a good performance against load and power reference disturbances as illustrated by its PR parameters, though it had a relatively poor performance against DC link voltage disturbance due to its control dependence on DC voltage dynamic. The number of control parameters in each technique is an important design factor to consider as it influences the control degrees of freedom. Therefore, VSM has the advantage of the additional flexibility provided by the virtual inertia parameter $(J)$.

An advanced black-start test should include transformer and cable models in addition to the considered static loads in this paper. That being said, the soft black-start test conducted here demonstrates, at a high level, that the four controllers are able to follow a reference voltage ramp while simultaneously

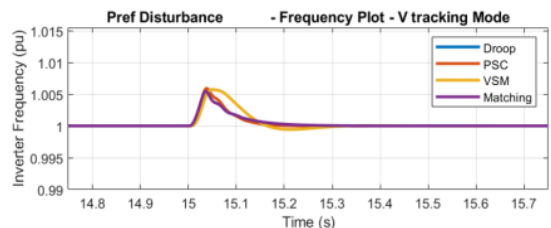

(a)

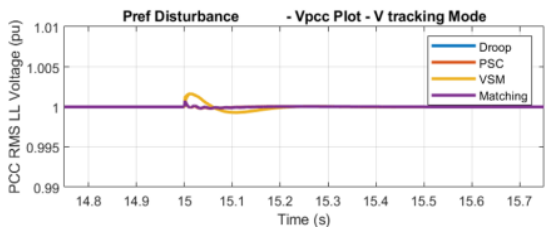

(d)

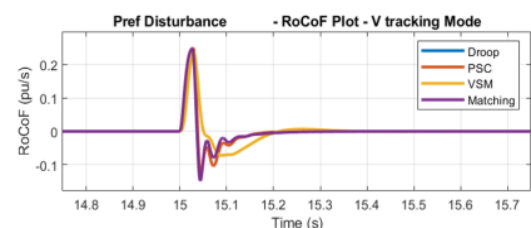

(b)

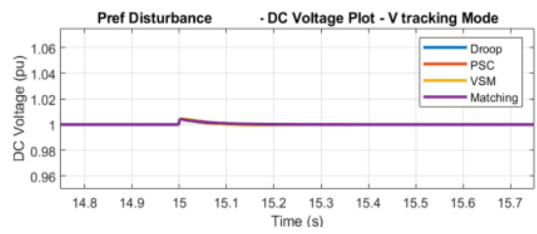

(e)

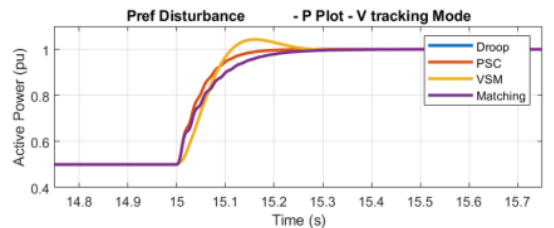

(c)

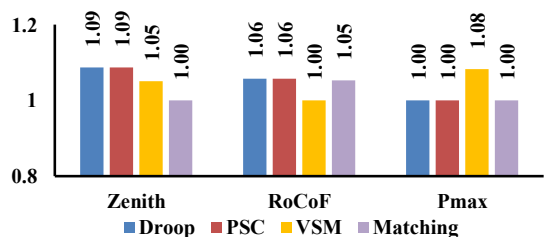

(f)

Fig. 8. GFCs control response to the power reference disturbance scenario. (a) Frequency plot, (b) RoCoF plot, (c) active power plot, (d) PCC voltage plot, (e) DC link voltage plot, (f) statistical GFC responses comparison. 

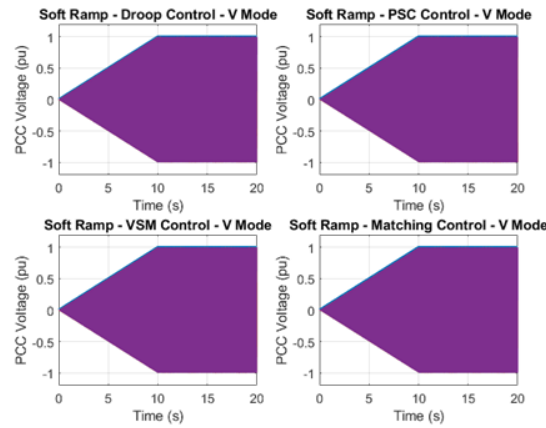

(a)
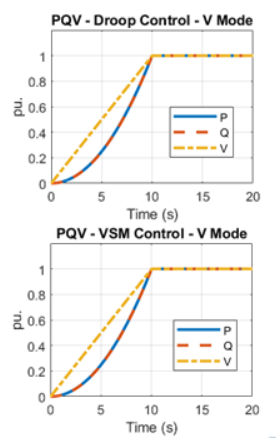

(b)
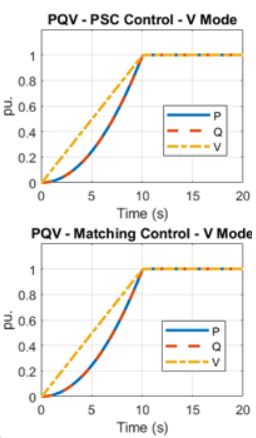

(b)

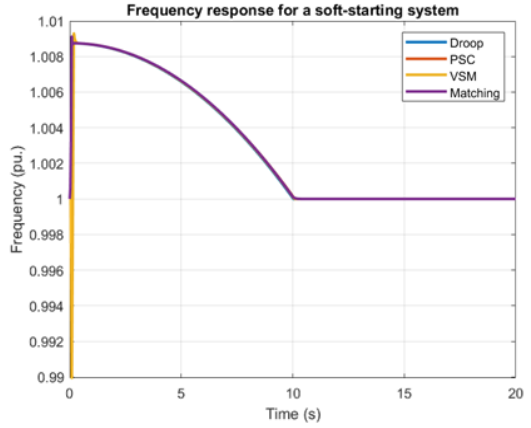

(c)

Fig. 9. GFCs response to the islanded soft-start scenario with $\mathrm{P}=35 \mathrm{MW}$ and $\mathrm{Q}=5 \mathrm{MVAr}$ ( (a): sinusoid voltage ramp, (b): $\mathrm{P}, \mathrm{Q}, \mathrm{V}$ ramp, (c): $f$ response.

supplying the required active and reactive power by the connected loads. This is an important step for a successful soft black-start implementation on a higher network-level.

\section{CONCLUSIONS}

This paper presented a performance evaluation of four GFC control techniques (Droop, PSC, VSM and Matching). The tested capabilities were chosen to reflect the controllers' robustness and flexibility against various disturbances. In addition, soft black-start compatibility was demonstrated for all the covered techniques. Simulation results indicate comparable performance for the four controllers against power and voltage disturbances. Matching control showed relatively poor performance against DC voltage reference disturbances. Droop and PSC performed almost identically due to their structural similarities with an overall good performance, and VSM demonstrated good response against different disturbances while also having an additional degree of freedom for additional improvement through its flexible virtual inertia parameter $(J)$. The outcomes of this study illustrate the potential and technical capabilities of GFCs and their suitability for black-start and grid-support applications. Recommended future work includes expanding the presented tests here to cover wider scenarios and network components.

\section{ACKNOWLEDGMENT}

This publication is supported by Iberdrola S.A. as part of its innovation department research activities. Its contents are solely the responsibility of the authors and do not necessarily represent the official views of Iberdrola Group.

\section{REFERENCES}

[1] R. H. Lasseter, Z. Chen, and D. Pattabiraman, "Grid-Forming Inverters: A Critical Asset for the Power Grid," IEEE Journal of Emerging and Selected Topics in Power Electronics, vol. 8, no. 2, pp. 925-935, 2020.

[2] A. Tayyebi, D. Groß, A. Anta, F. Kupzog, and F. Dörfler, "Frequency Stability of Synchronous Machines and Grid-Forming Power Converters," IEEE Journal of Emerging and Selected Topics in Power Electronics, vol. 8, no. 2, pp. 1004-1018, 2020.

[3] W. Du et al., "A Comparative Study of Two Widely Used Grid-Forming Droop Controls on Microgrid Small-Signal Stability," IEEE Journal of Emerging and Selected Topics in Power Electronics, vol. 8, no. 2, pp. 963-975, 2020.

[4] "Black Start from Non-Traditional Generation Technologies," National Grid, 2019.
[5] G. Patsakis, D. Rajan, I. Aravena, J. Rios, and S. Oren, "Optimal Black Start Allocation for Power System Restoration," IEEE Transactions on Power Systems, vol. PP, pp. 1-1, 05/22 2018, doi: 10.1109/TPWRS.2018.2839610.

[6] J. Matevosyan et al., "Grid-Forming Inverters: Are They the Key for High Renewable Penetration?," IEEE Power and Energy Magazine, vol. 17, no. 6, pp. 89-98, 2019.

[7] J. Li, H. You, J. Qi, M. Kong, S. Zhang, and H. Zhang, "Stratified Optimization Strategy Used for Restoration With Photovoltaic-Battery Energy Storage Systems as Black-Start Resources," IEEE Access, vol. 7, pp. 127339-127352, 2019.

[8] Z. S. Joukhah, "Operation of HVDC converters for transformer inrush current reduction," $\mathrm{PhD}$ Thesis, Electrical Engineering, Polytechnic University of Catalonia, 2017.

[9] M. Aten, R. Shanahan, F. Mosallat, and S. Wijesinghe, "Dynamic Simulations of a Black Starting Offshore Wind Farm Using Grid Forming Converters," 18th Wind Integration Workshop, Dublin, 2019.

[10] O. Schomann, "Experiences With Large Grid-Forming Inverters on Various Island and Microgrid Projects," in Hybrid Power Systems Workshop, Crete, 2019.

[11] M. Ashabani, F. D. Freijedo, S. Golestan, and J. M. Guerrero, "Inducverters: PLL-Less Converters With Auto-Synchronization and Emulated Inertia Capability," IEEE Transactions on Smart Grid, vol. 7, no. 3, pp. 1660-1674, 2016.

[12] D. Pan, X. Wang, F. Liu, and R. Shi, "Transient Stability of VoltageSource Converters With Grid-Forming Control: A Design-Oriented Study," IEEE Journal of Emerging and Selected Topics in Power Electronics, vol. 8, no. 2, pp. 1019-1033, 2020.

[13] A. Khan, M. hosseinzadehtaher, M. B. Shadmand, S. Bayhan, and H. Abu-Rub, "On Stability of Power Electronics Dominated Grid," IEEE Industrial Electronics Magazine, vol. 14, no. 2, 2020.

[14] L. Zhang, L. Harnefors, and H. Nee, "Power-Synchronization Control of Grid-Connected Voltage-Source Converters," IEEE Transactions on Power Systems, vol. 25, no. 2, pp. 809-820, 2010.

[15] A. Alassi, S. Bañales, O. Ellabban, G. Adam, and C. MacIver, "HVDC Transmission: Technology Review, Market Trends and Future Outlook," Renewable and Sustainable Energy Reviews, vol. 112, pp. 530-554, 2019.

[16] M. Ahmed, L. Meegahapola, A. Vahidnia, and M. Datta, "Analyzing the Effect of X/R ratio on Dynamic Performance of Microgrids," in 2019 IEEE PES Innovative Smart Grid Technologies Europe (ISGT-Europe), 29 Sept.-2 Oct. 2019 2019, pp. 1-5.

[17] Q. Zhong and G. Weiss, "Synchronverters: Inverters That Mimic Synchronous Generators," IEEE Transactions on Industrial Electronics, vol. 58 , no. 4, pp. 1259-1267, 2011.

[18] C. Arghir, T. Jouini, and F. Dörfler, "Grid-forming control for power converters based on matching of synchronous machines," Automatica, vol. 95, pp. 273-282, 2018.

[19] J. Fang, H. Li, Y. Tang, and F. Blaabjerg, "Distributed Power System Virtual Inertia Implemented by Grid-Connected Power Converters," IEEE Transactions on Power Electronics, vol. 33, no. 10, pp. 8488-8499, 2018.

[20] C. Arghir and F. Dörfler, "The Electronic Realization of Synchronous Machines: Model Matching, Angle Tracking, and Energy Shaping Techniques," IEEE Transactions on Power Electronics, vol. 35, no. 4, pp. 4398-4410, 2020. 